\title{
The Biochemical Effect of Smoking on the Response to Chemotherapy
}

\begin{abstract}
OANA MIRON ${ }^{1}$, ADINA MAGDALENA TURCANU ${ }^{1,2 *}$, BOGDAN MIHNEA CIUNTU ${ }^{3 *}$, VLADIMIR POROCH ${ }^{1}$, DANIEL TIMOFTE ${ }^{1}$
${ }^{1}$ Grigore T. Popa University of Medicine and Pharmacy, Faculty of Medicine, 16 Universitatii Str., 700115, lasi, Romania

${ }^{2}$ Clinical Hospital of Pneumology, 30 Doctor losif Cihac Str., 700115, Iasi, Romania

${ }^{3}$ Grigore T. Popa University of Medicine and Pharmacy, Department of Radiology, 16 Universitatii Str., 700115, Iasi, Romania

Improvements in early detection of cancer have led to an important decrease in mortality rates of cancer. Given the increased incidence rates and decreased mortality rates, the number of patients surviving cancer is rapidly increasing. Although cancer patients face many physical and psychological symptoms, they also continue to engage in poor health behaviors at rates similar to those of the general-healthy population. The prime example of such unhealthy behavior is smoking. The reports show that smoking rates at the time of diagnosis of cancer vary from $10 \%$ to $95 \%$. Our study analyzed how the smoking status influenced the outcome of chemotherapy of 249 patients suffering from various forms of cancer. Our statistical analysis showed that patients who smoked had a significant different response to chemotherapy compared to their nonsmoking peers. This meant that in our sample of 149 cancer suffering patients, individuals who did not smoke had a significant better chance of a partial positive response after chemotherapy compared to patients who smoked regularly. Therefore, tobacco smoking is an adverse prognostic factor associated with a resistance to chemotherapy. These results are important given the fact that cancer patients already face a combination of unpleasant symptoms related to their disease but also from the side effects of their treatment. Uncovering the exact mechanisms through which smoking is affecting the outcome of chemotherapy may help in increasing the quality of life, the symptom burden or the final outcome of chemotherapy.
\end{abstract}

Keywords: cancer, chemotherapy, tobacco, smoking, side effects, biochemical mechanisms, cancer treatment.

In the last decades certain improvements in early detection of cancer plus the progress made in the treatment of this disease have made possible an important observed decrease in mortality rates of cancer [1]. Given this context, increased incidence rates and decreased mortality rates, the number of patients surviving cancer is rapidly increasing. For example, it has been reported that in 2017 the number of cancer survivors living in the United States was close to 12 million [2]. Furthermore, the number of cancer survivors is expected to increase, as studies show that $68 \%$ of the 1.5 million diagnosed with cancer in 2018 in the United States only, are expected to survive in an average of 5 years [3]. On the other hand, the chemotherapy and radiotherapy destroy malignant cells and improve survival rates in cancer patients, but most often also damage healthy tissues, resulting in undesired side effects [4]. Therefore, as the number of cancer survivors inflates, it is increasingly important to identify what healthy/unhealthy habits may positively influence their response to chemotherapy.

Therefore, cancer patients face a unique combination of health problems related to their disease but also from the side effects of their treatment. In the literature, chemotherapy is associated with both short-term and longterm side effects. Regarding the side effects of chemotherapy, cancer- related fatigue is one of the most common reaction and studies show that this symptom is experienced by up to $99 \%$ of the patients. This side effect is described as overwhelming fatigue followed by a low capacity for physical and mental work which is not relieved by any amount of relaxation [5]. Furthermore, it is problematic that this exhaustion was found to persist in cancer survivors for years after treatment completion [6]. The data shows that no less than $35 \%$ of cancer patients still presented persistent fatigue symptoms 5 years after treatment completion [7].

How ever, the most unpleasant and feared side effects are chemotherapy-induced nausea and vomiting [8]. Even if vomiting is well controlled by antiemetic medication nausea remains a problematic side effect of cancer treatment [9]. Another important side effect of chemotherapy is represented by sleep disruption. The results available in the literature show that cancer treatment patients report three times more sleep disruptions compared to the general population [10].

Another important side effect that should be mentioned here is the so called chemobrain. Chemobrain is described as cognitive impairments such as memory problems and having difficulties concentrating. [11]. Short-term chemobrain has been shown in the available studies to occur in up to $75 \%$ of patients who undergo chemotherapy [12]. Furthermore, long-term cognitive difficulties associated with chemobrain have also been reported. The available literature shows that up to $45 \%$ of cancer patients are reporting cognitive deficits many years after the completion of chemotherapy [13].

Another medical concern regarding the patients who undergo chemotherapy is depression. The studies show that depression rates exceed $50 \%$ in cancer patients. This is an alarming percent compared to $4 \%-17 \%$ prevalence of depression symptoms in general population [14]. In addition, studies show that in cancer patients, depression frequently coexists with anxiety and pain. This is problematic because it has been shown that these symptoms are associated with prolonged recovery and poor outcomes of chemotherapy [15]. In addition, depression and anxiety have also been linked to treatment

*email: adinagheorghita@yahoo.com, bogdanmciuntu@yahoo.com 
interruptions and dosage reductions, resulting in lower efficacy of chemotherapy [16].

As we mentioned above, although cancer patients face significantly more physical and psychological symptoms compared to their healthy peers, they also continue to engage in poor health behaviors at rates similar to those of the general population. The prime example of such unhealthy behavior is smoking. The reports show that smoking rates at the time of diagnosis of cancer vary from 10 to $95 \%$, depending on the cancer site [17]. However, newly diagnosed cancer patients quit smoking at different rates, depending by cancer site. These rates range from $5 \%$ for breast cancer cases to $60 \%$ for lung cancer patients [18]. In addition, as before mentioned, the available reports show little difference in smoking prevalence between cancer patients and the general population at all ages. Therefore, at all ages, smoking has a prevalence of $20 \%$ in cancer patients and of $24 \%$ in the general population [19].

Although smoking while suffering from cancer is associated with a greater mortality and more treatmentrelated complications, there is little research on the effect of smoking on chemotherapy response. The primary aim of this study was to determine the effect of smoking on chemotherapy response in patients undergoing treatment for cancer.

\section{Experimental part \\ Methods}

The study was conducted on 249 patients suffering from various forms of cancer who received chemotherapy at the Regional Institute of Oncology lasi. Smoking status was measured with a simple, single question, Are you currently a smoker (yes, no)?

\section{Statistical Analyses}

For descriptive statistics One Way Anova was used were used to analyze the differences between smokers and nonsmokers for the dependent variable response to chemotherapy. The response to chemotherapy variable had 4 levels: 1- Partial response, 2- stationary disease, 3progresive disease, 4- complete response. Unfortunately, none of the patients presented a complete response to chemotherapy, so this level of the variable was eliminated from our statistical analysis.

Furthermore, we also wanted to discover if any of the demographic variables can influence the result of chemotherapy. Therefore, other One Way Anova was conducted to analyze the difference between men and women regarding the response to chemotherapy. Lastly, a One Way Anova was also conducted to analyze the possible differences between patients from rural areas and patients form urban areas.

\section{Results and discussions}

A one way between subjects ANOVA was conducted to compare the chemotherapy response in patients with a smoking habit compared to non-smoking patients.

The results of the statistical analysis showed a significant difference regarding the response to chemotherapy in the smokers group $(M=2.126)$ compared to the non-smokers group ( $M=1.859) ; F(1,245)=6.594, p=0.011$ (Fig.1).

Next we analyzed if other demographic variables might influence the chemotherapy response. Therefore, a one way betw een subjects ANOVA was conducted to compare the chemotherapy response in women compared to men.

The results of the statistical analysis showed a nonsignificant difference regarding the response to chemotherapy in the women group ( $M=1.907)$ compared

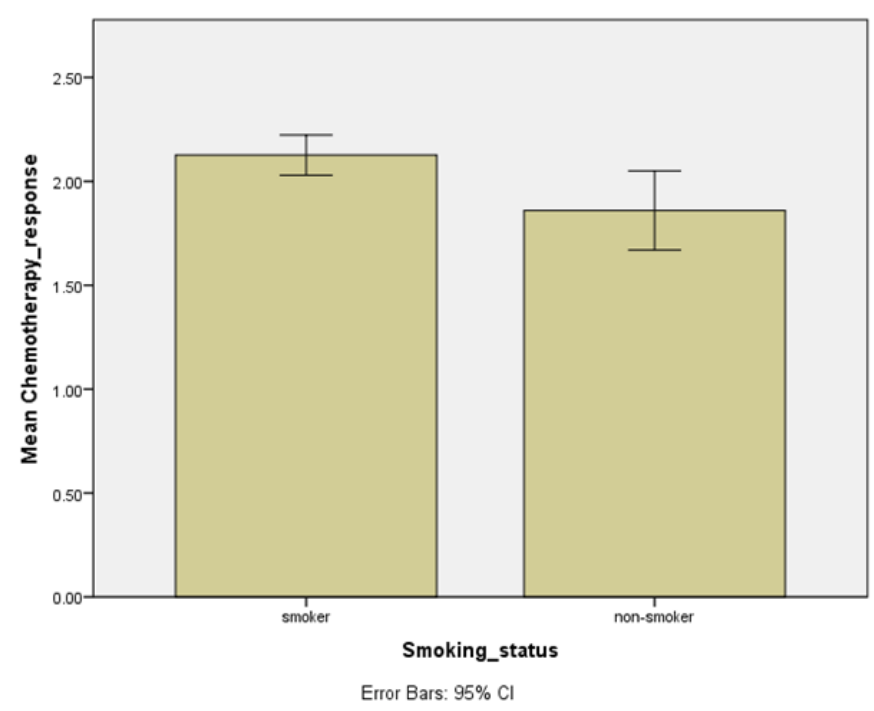

Fig.1. The response to chemotherapy for smokers compared with non-smokers.

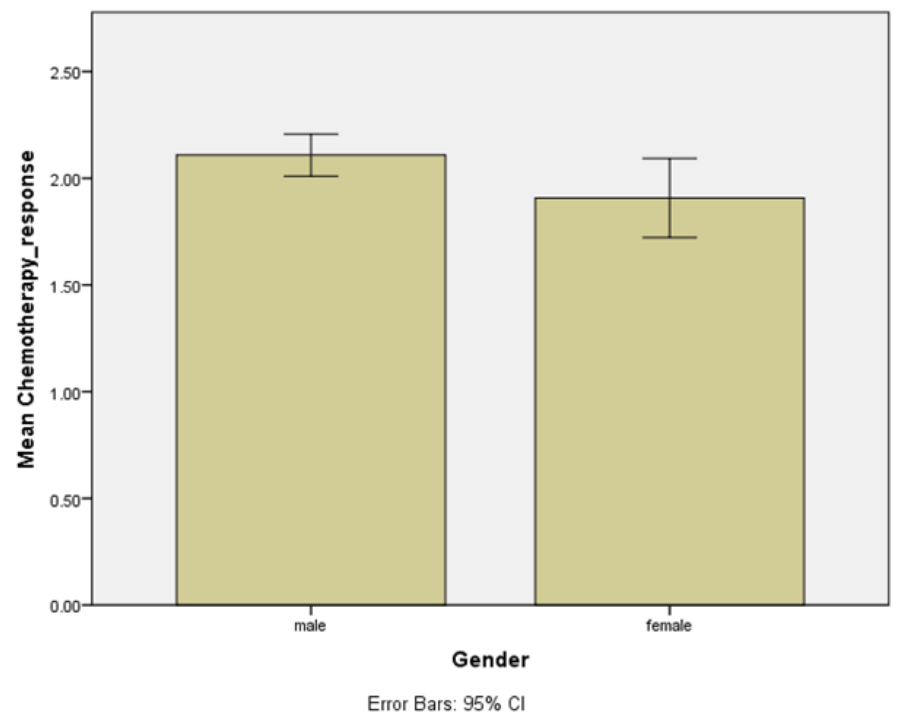

Fig.2. The response to chemotherapy for males compared with females.

to the men group $(M=2.108) ; F(1,245)=3.576, p=0.060$ (Fig.2).

For the next demographic variable, a one way between subjects ANOVA was conducted to compare the chemotherapy response in patients from rural areas with patients from urban areas.

The results of the statistical analysis showed a nonsignificant difference regarding the response to chemotherapy in the rural group ( $M=2.054)$ compared to the urban group $(M=2.070) ; F(1.245)=0.029, p=0.866$ (Fig.3).

The importance of our study comes from the fact that unhealthy behaviors such as cigarette smoking during cancer treatment may have an impact on treatment outcomes for cancer patients. The results of our study are in concordance to those found in the available literature. Our statistical analysis showed that patients who smoked had a significant different response to chemotherapy compared to their nonsmoking peers. This meant that in our sample of 149 cancer suffering patients, individuals who did not smoke had a significant better chance of a partial positive response after chemotherapy compared to patients who smoked regularly. In addition, individuals who declared that they have a smoking habit had a better chance of a progressive disease response after the chemotherapy treatment. These results are not surprising 


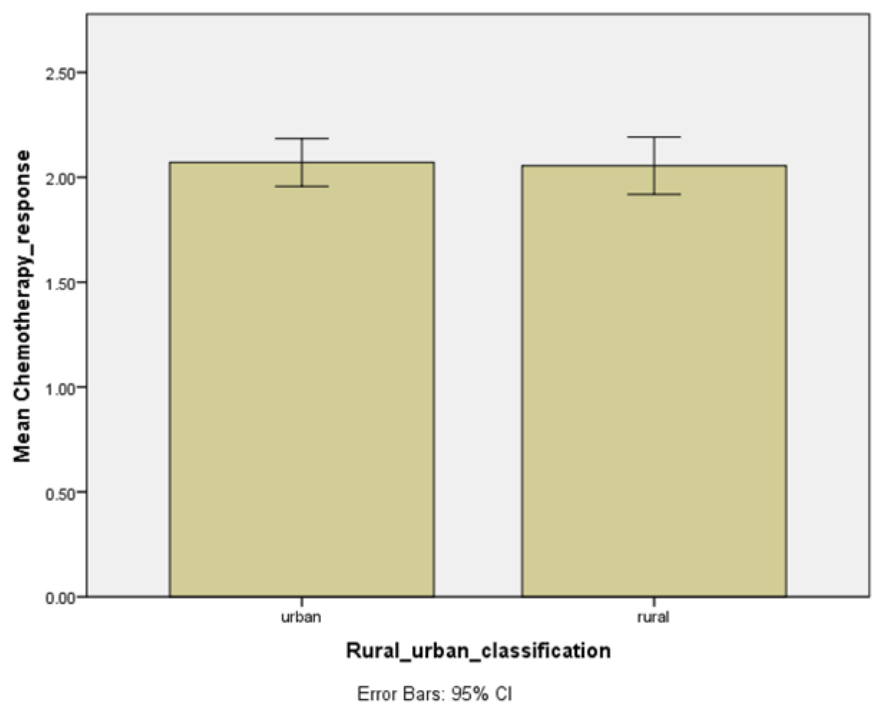

Fig.3. The response to chemotherapy for patients with an urban origin compared with patients from a rural area.

and are similar with previous studies. The reported results of the available literature shows that patients who smoke throughout cancer treatment have a worse response to chemotherapy and a significantly lower survival rate when compared to individuals who do not smoke [20-22]. Furthermore, patients who smoked during chemotherapy have been found to present a significantly higher risk of developing second primary tumors and other various treatment-related complications [23, 24]. The negative effects of smoking during cancer treatment do not end here. Available reports show that cancer patients who smoke during chemotherapy present a lower quality of life when compared to cancer patients who do not smoke [25]. The primary explanation of the low quality of life reported by the smokers is that many quality of life domains such as the physical, functional, and social-emotional ones are directly related to chemotherapy specific side effects. This side effects are the before mentioned fatigue, pain, depression, anxiety and the symptom burden itself.

These results show that smoking during cancer treatment is associated with a lower probability of a positive partial response after chemotherapy. The results of our experiment are consistent with other studies that also found that smoking during treatment leads to a lower chance of a positive response after cancer treatment.

The responsible biological mechanisms that might account for this association found between smoking and cancer treatment response have yet to be clarified. Some authors proposed the hypothesis that the same mechanisms that lead to these negative chemotherapy responses also increase symptom burden. For example, some well-known side effects, such as physical exhaustion, depression, anxiety and insomnia, may be caused by the occurrence of cellular damage. This cellular damage induces local inflammation, modifies hormone levels, and in this way might therefore interfere with the normal circadian rhythm of individuals.

In addition, in our study we wanted to know if other sociodemographic potential confounding variables such as biological gender or rural/ urban classification might also have a significant effect on the response to chemotherapy in our sample of patients. The results of these analyses are statistically non-significant. Our statistical analysis showed that there were no significant differences between men and women, or patients from rural areas and patients from urban areas in regards to their positive, stationary or negative response to chemotherapy.

Therefore, tobacco smoking is an adverse prognostic factor associated with a resistance to chemotherapy in our sample of patients. Smoking has also been demonstrated to be a negative factor to chemotherapy response in other studies. For example, in an important study, it has been showed that an association exists between smoking during cancer treatment and poor outcome. The authors of this study attributed this effect to acute hypoxia. However, the same authors obtained statistically non-significant results in a follow-up confirmatory study [26]. Accordingly, the role of smoking as a negative prognostic factor during various cancer treatments remains unclear and deserves further examination. Other possible mechanism for this association apart from hypoxia may be a p53 mutation caused by smoking tobacco that was found in various researches to be correlated with a resistance to apoptosis [27].

Thus, a negative effect of smoking on chemotherapy response has been demonstrated; however, the possible mechanisms of this association remain yet to be revealed. Other proposed mechanism refers to the fact that smoking increases blood carboxyhemoglobin, producing hypoxia by decreasing the loading capacity of oxygen by the hemoglobin [28]. However, the level of carboxyhemoglobin returns to normal ranges 8 hours after the smoking has ceased [29]. If acute hypoxia is implicated in negative response to chemotherapy, this may be of interest to patients who refuse to quit smoking during cancer treatment, as these individuals may receive the chemotherapy in the morning after abstaining from smoking for the night before.

These aspects could be of also correlated to some degree to our group latest interests in combinations that might exist between smoking status, exercise performing and oxidative stress status modifications [30-33].

\section{Conclusions}

The results of the present study show that smoking is negatively influencing the response of chemotherapy of cancer suffering patients. These results are important given the fact that cancer patients already face a combination of unpleasant symptoms related to their disease but also from the side effects of their treatment. Uncovering the exact mechanisms through which smoking is affecting the outcome of chemotherapy may help in increasing the quality of life, the symptom burden or the final outcome of cancer treatment.

\section{References}

1. WINGO, P., CARDINEZ, C., LANDIS, S., et al. Cancer., 97, nr. 12, 2003, p. 3133.

2. YOU, W., HENNEBERG, M., Evol. Apl., 11, nr. 2, 2018, p. 140.

3. JEMAL, A., SIEGEL, R., XU, J., et al. Cancer J. Clin., 60, 2010, p. 277. 4. REDD, W., JACOBSEN, P., ANDRYKOWSKI, M., Recent Results Cancer Res., 115, 1989, p. 272.

5. RYAN, J., CAROLL, J., RYAN, E., et al., The Oncologist, 12, nr. 1, 2007, p. 22.

6. BOWER, J., GANZ, P., DESMOND, K., etal., Cancer, 106, 2006, p. 751. 7. SERVAES, P., GIELISSEN, M., VERHAGEN, S., et al., Psychooncology, 16,2007, p. 787.

8. HESKETH, P., N. Engl. J. Med., 358, 2008, p. 2482.

9. ETTINGER, D., ARMSTRONG, D., BARBOUR, S., et al., J . Natl. Compr. Canc. Netw., 7, 2009, p. 572.

10. PALESH, O., ROSCOE, J., MUSTIAN, K., et al., J. Clin. Oncol., 28, 2010, p. 292. 
11. SCHAGEN, S., VAN DAM, F., MULLER M., et al., Cancer, 85, 1999p. 640.

12. AHLES, T., SAYKIN, A., FURSTENBERG, C., et al., J. Clin. Oncol., 20, 2002, p. 485.

13. WEIS, J., POPPELREUTER, M., BARTSCH, H., Psychooncology, 18, 2009, p. 775

14. KIM, Y., HICKOK, J., MORROW, G., J. Pain. Symptom Manage, 32, 2006, p. 311.

15. DEROGATIS, L., MORROW, G., FETTING, J., et al., JAMA, 249, 1983, p. 751.

16. REICH, S., Cancer Nurs., 7, 1984, p. 59.

17. DEMARK-WAHNEFRIED, W., PETERSON, B., MCBRIDE, C., et al., Cancer, 88, 2000, p. 674.

18. OSTROFF, J., JACOBSEN, P., MOADEL, A. et al., Cancer, 75, 1995, p. 569.

19. DEMARK-WAHNEFRIED, W., AZIZ, N., ROWLAND, J., et al., J. Clin. Oncol., 23, 2005, p. 5814.

20. DUFFY, S., RONIS, D., MCLEAN, S. et al., J. Clin. Oncol., 27, 2009, p. 1969.

21. BROWMAN, G., WONG, G., HODSON, I., et al., N. Engl. J. Med., 328, 1993, p. 159.

22. FORTIN, A., WANG, C., VIGNEAULT, E., Int. J. Radiat. Oncol. Biol. Phys., 74, 2009, p. 1062.

23. KHURI, F., LEE, J., LIPPMAN, S., et al., J. Natl. Cancer Inst., 98, 2006, p. 441.

24. EIFEL, P., JHINGRAN, A., BODURKA, D., et al., J. Clin. Oncol., 20, 2002, p. 3651.

25. GRITZ, E., CARMACK, C., DE MOOR, C., et al., J. Clin. Oncol., 17, 1999, p. 352.

26. BROWMAN G., MOHIDE, E., WILLAN, A., et al., Head Neck, 24, 2002, p. 1031.
27. LILOGLOU, T., SCHOLES, A., SPANDIDOS, D., VAUGHAN, E., JONES, A., FIELD J., Cancer Res., 57, 1997, p. 4070.

28. JENSEN, J., GOODSON, W., HOPF, H., HUNT, T., Arch. Surg., 126, $1991,1131$.

29. KAMBAM, J., CHEN, L., HYMAN, S., Anesth. Analg., 65, 1986, p. 1186.

30. TROFIN, F., CIOBICA, A., HONCERIU, C.,COJOCARU, SI.,STOICA, B., COJOCARU, D.,CIORNEA, E.,TIMOFTE, D., Modulatory effects of vitamin $C$ on the relation between physical exercising and oxidative stress at young smokers, ROMANIAN BIOTECHNOLOGICAL LETTERSVolume: 22 Issue: 2 Pages: 12439-12447 Published: MARAPR 2017

31. ARCAN, O; BILD, W.,CIOBICA, A., SERBAN, DN., ANTON, E., PETRARIU, F.,TIMOFTE, D., NASTASA, V., Angiotensin-(1-7) intracerebroventricular administration generates nociceptive effects in hot-plate task and decreased oxidative stress in the temporal lobe, ROMANIAN BIOTECHNOLOGICAL LETTERS Volume: 19, Issue: 5, Pages: 9763-9771 Published: SEP-OCT 2014

32. TIMOFTE, D.,TOARBA, C.,HOGAS, S.,COVIC, A.,CIOBICA, A.,CHIRITA, R., LEFTER, R., ARHIRE, L., ARCAN, O., ALEXINSCHI, O., SERBAN, D., GRAUR, M., POROCH, V., The Relevance of Oxidative Stress Status in Type 2 Diabetes and the Chronic Consumption of Alcohol, ROMANIAN BIOTECHNOLOGICAL LETTERS Volume: 21 Issue: 1 Pages: 11246-11253 Published: JAN-FEB 2016 33. STOIAN, A.P., MITROFAN, G., COLCEAG, F., SUCEVEANU, A.I., HAINAROSIE, R., PITURU, S., DIACONU, CC., TIMOFTE, D., NITIPIR, C., POIANA, C., SERAFINCEANU, C., Oxidative Stress in Diabetes A model of complex thinking applied in medicine, Rev.Chim. (Bucharest), 69, no.9, 2018, p.2515-2519

$\overline{\text { Manuscript received:22.11.2018 }}$ 